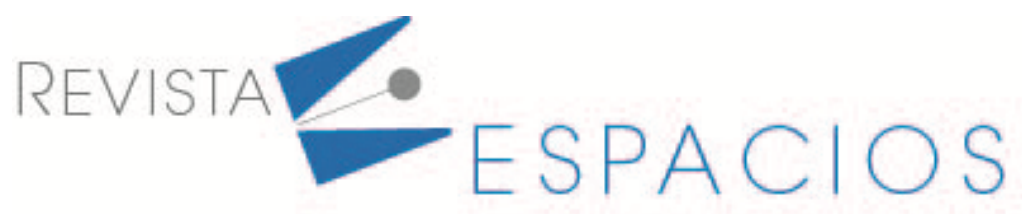

\title{
Cultura organizacional en el proceso de gestión de la calidad educativa
}

\section{Organizational culture in the educational quality management process}

\author{
SERRANO-POLO, Edgardo ${ }^{1}$ \\ GONZÁLEZ-DIAZ, Romel R. ${ }^{2}$ \\ OVALLES-TOLEDO, Luiz V. ${ }^{3}$ \\ RUIZ-CORRALES, Cristina M. ${ }^{4}$
}

\begin{abstract}
Resumen
Este estudio analiza las diferentes manifestaciones de la cultura organizacional en el proceso de gestión de la calidad educativa en instituciones públicas de Colombia. Se aplicó un cuestionario a 10 rectores y 40 docentes y administrativos. Los principales hallazgos hacen alusión a la existencia de discrepancia en cuanto a la relación del clima organizacional, destacando los valores, las interacciones y la productividad en el proceso de gestión de la calidad educativa.

Palabras clave: cultura organizacional, gestión escolar, calidad educativa, instituciones educativas.
\end{abstract}

\begin{abstract}
This study analyzes the different manifestations of organizational culture in the educational quality management process in public institutions in Colombia. A questionnaire was applied to 10 principals and 40 teachers and administrators. The main findings allude to the existence of discrepancy regarding the relationship of the organizational climate, highlighting the values, interactions and productivity in the educational quality management process.

key words: organizational culture, school management, educational quality, educational institutions.
\end{abstract}

\section{Introducción}

Todos los fenómenos sociales donde se mueven las actividades humanas, a través de la globalización, generan mayores exigencias en crecimiento de la economía y competitividad, que se perciben desde las escuelas hasta las grandes corporaciones; en este sentido, se acrecientan las demandas de mayor calidad de servicios y bienes conducentes al éxito organizacional (Arboleda \& Díaz, 2017; Díaz \& Ramos, 2019).

Generalmente, se plantean a las organizaciones como la expresión del ámbito cultural, que atiende a los permanentes cambios de la dinámica de la sociedad en los aspectos económico y tecnológico. De manera que la naturaleza de estos mecanismos refleja un marco de valores, creencias, ideas, sentimientos y voluntades de una comunidad institucional que definen la cultura (Touriñán López, 2019).

\footnotetext{
${ }^{1}$ Subdirector del Centro Internacional de Investigaciones y Desarrollo (CIID). Correo electrónico de contacto: asesor@ciid.com.co

2 Director del Centro Internacional de Investigaciones y Desarrollo (CIID). Correo electrónico de contacto: director@ciid.com.co

${ }^{3}$ Coordinador General de Investigación y Postgrado de la Facultad de Contaduría y Administración. Universidad Autónoma de Sinaloa. Correo electrónico de contacto: luiz.ovalles@uas.edu.mx

${ }^{4}$ Líder de investigación SENOVA-Montería. SENA.Correo electrónico de contacto: cristinaruiz@sena.gov.co
} 
No obstante, haciendo un alto en el área educativa, la cultura organizacional se convierte en un aspecto muy poco abordado, cuando se piensa en el desarrollo de las actividades académicas que promuevan la gestión de la calidad educativa (Maldonado \& del Pilar, 2019). Dado a que sólo se involucran aspectos pedagógicos para la planificación y ejecución de las clases, apartando los compromisos laborales, el liderazgo, los valores, la pertinencia, la comunicación y la productividad entre los miembros escolares; por tanto, no se refleja lo que se piensa y se opera dentro de la organización que intervienen de un modo directo sobre el rendimiento académico, capacidades, actitudes de los estudiantes y otro fines de la institución (Freire, Jácome, \& Yandún, 2019).

Precisamente en el panorama educativo colombiano se observa, que al evaluar las competencias básicas de los estudiantes, en pruebas Saber y en las pruebas internacionales como PISA, se han obtenido resultados muy preocupantes (Carrascal \& Giraldo, 2019). En este mismo sentido, los rectores, los docentes y encargados de las escuelas denuncian debilidades en la infraestructura, en los recursos, en los cambios imprevistos de políticas de Estado, que permiten aplicar bien un programa cuando se exigen otros sin esperar los resultados del anterior, lo que trae confusión, apatía e indiferencia de personal.

La problemática se agudiza cuando una institución no maneja las manifestaciones de una cultura organizacional que se identifique con su misión, visión, objetivos y metas claras, que conlleve a un trabajo mancomunado para solventar las dificultades y encontrar consenso que den respuestas desde la propia organización (Ovalles-Toledo, Martínez, Arrieta, \& Aguas, 2016). Asimism o, existen debilidades en los procesos de supervisión, control, una exigencia equitativa ante los planes y programas gubernamentales del Ministerio de Educación Nacional.

En virtud de la realidad mencionada es particularmente palpable, en instituciones educativas a nivel primaria y secundaria, donde el trabajo interorganizacional, requiere de una gestión con mayores fuerzas activas desde los rectores con su personal, para trabajar eficientemente por sus estudiantes, dirigiendo su accionar al mejoramiento de la calidad educativa.

Atención de las razones antes mencionadas, surge la idea de desarrollar esta investigación, para así aportar conocimientos sobre el estudio de la cultura organizacional en pro de la calidad educativa, y así contribuir desde la perspectiva del rigor científico. Es por ello, que la investigación tiene como objetivo determinar las manifestaciones de la cultura organizacional en el proceso de gestión de la calidad educativa en instituciones públicas de Colombia, particularmente en el Distrito Turístico, Cultural e Histórico de Santa Marta.

\subsection{Fundamentación Teórica}

\section{Cultura Organizacional}

La cultura organizacional constituye un marco de referencia para cada uno de los sujetos que conforman la organización con normas y valores de comportamiento para la conducción de sus acciones, de manera que exista una línea orientadora de los patrones y actitudes conductuales.

Desde un sentido etimológico la palabra cultura viene del latín cultura y este de cultus, cultivo cultivado, participio de colere, cultivar. El sufijo latino ura, significa actividad, la actividad resultante de una acción. Para Echeverría (2019) la cultura es un patrón de supuestos básicos compartidos que el grupo aprende en la medida que resuelve sus problemas de adaptación externa e integración interna, que los ha trabajado lo suficiente para ser considerados como válidos y, por lo tanto, dignos de ser enseñados a los nuevos miembros como la forma correcta de percibir, pensar y sentir en relación con esos problemas.

Por otro parte, Mead (2019) expone que la cultura es la conducta convencional de una sociedad, e influye en todas sus acciones a pesar de que rara vez esta realidad penetra en sus pensamientos conscientes. El autor asegura que la gente asume con facilidad su cultura, además, que ésta le da seguridad y una posición en cualquier entorno donde se encuentre. En este sentido, Steiner (2020), coincide al denominar a la cultura organizacional 
como una percepción que comparten todos los miembros de la Organización: Sistema de significados compartidos.

Obviamente la cultura organizacional se observa de forma relativa, por lo que los sujetos crean patrones de comportamiento, aprenden, y desarrollan actividades, se transforman y evolucionan. Desde esta perspectiva se plantean aspectos del entorno social, que, exigen capacidades y competencias en los gerentes ante la eventualidad de la dinámica social.

Un significativo concepto de la cultura organizacional lo expone García (2020), al considerar que es una manera como cada organización aprendió a tratar su ambiente y sus socios; es una mezcla compleja de presuposiciones, creencias, comportamientos, historias, mitos, metáforas y otras ideas que, en conjunto, representan la manera como funciona y trabaja una organización.

Efectivamente los señalamientos esgrimidos coinciden con Leonard, Pérez, and Domínguez (2020) quienes señalan que la cultura es un vínculo social que ayuda a mantener unida a la organización al proporcionar normas adecuadas a lo que debe hacer y decir el personal; relacionados con los elementos presentes e integrados en la cultura organizativa; la cual, es indispensable identificar y medir para determinar si ameritan cambiarlos o reforzarlos, pues son compartidos por los integrantes de la organización.

Basado en estas premisas la cultura organizacional en la perspectiva dinámica, como la referencia necesaria para toda organización burocrática en tanto pone de manifiesto la cuestión de la toma de decisión que involucra una serie de factores, los cuales pueden ser percibidos con más facilidad que otros; además de presentar aspectos formales e informales, lo que da origen a un todo complejo debido a sus características ocultas o visibles.

\section{Funciones de Cultura Organizacional}

Para Álvarez (2019), las empresas deberían buscar un consenso entre trabajadores y dirección respecto a los hitos culturales: misión, objetivos, medios para conseguir objetivos, evaluación de objetivos y estrategias de lo que hay que hacer cuando las cosas van mal.

Entre las funciones que se le atribuye a la cultura de las organizaciones, se resumen las siguientes formas:

-En primer lugar tiene un papel de definición de límites; es decir, crea diferencias entre una organización y las demás.

-En segundo lugar, conlleva un sentido de identidad para los miembros de la organización.

-En tercer lugar, la cultura facilita la generación del compromiso con algo más grande que el interés personal del individuo.

-En cuarto lugar, mejora la estabilidad del sistema social. Por último la cultura sirve como mecanismo de control y de sensatez que guía y modela las actitudes y el comportamiento de los empleados.

\section{Manifestaciones de Cultura Organizacional}

Cuando entran en acción la cultura y las manifestaciones culturales, emerge una dinámica de realimentación, dado que la cultura se refleja en las manifestaciones culturales y las manifestaciones culturales a su vez nutren y enriquecen a la cultura. Las manifestaciones culturales son las expresiones o productos de un sistema cultural que reflejan las creencias y los valores básicos de sus miembros. Burke (2020) quien reformula el término sistema cultural como aquel que se integra por el conjunto de valores y creencias que comparten las personas que pertenecen a él, y por las múltiples formas en que se manifiestan dichos valores y creencias, modelo que permitiría considerar sistemas culturales a mayor variedad de agrupaciones humanas. 
En una cultura organizacional, de acuerdo con (Álvarez, 2019; Carrillo-Punina, 2019; Valdeyglesias, Gerty, Chávez Muñoz, \& Ortiz Castillo, 2019) entre otros autores, existen una serie de indicadores y manifestaciones culturales que caracterizan el sistema de presunciones básicas de la organización. Los indicadores de la cultura organizacional están conformados, entre otras, por las siguientes manifestaciones: a) Clima organizacional, b) Valores organizacionales, c) Las interacciones y d) Productividad.

a) Clima organizacional: es la manifestación más visible de la cultura; entre los aspectos que comprometen esta manifestación están por ejemplo: el liderazgo, la satisfacción, el reconocimiento y el ambiente físico. El Liderazgo, un aspecto fundamental del clima organizacional, entendiéndose de acuerdo con Velazquez Valadez (2019) como el ambiente y las condiciones en donde opera una organización pueden requerir competencias de liderazgo que deben ser evaluadas en los gerentes, porque aglutinan elementos y enfoques sobre la efectividad y pertinencia de roles de acuerdo con las características del ambiente y de la organización. Argumentan que la gerencia efectiva requiere la capacidad de usar diversos modelos de gestión, aun cuando éstos se basen en diferentes enfoques desarrollados en la teoría organizacional, lo que conlleva a que el liderazgo sea simple y lógico, dinámico y sinergético, o bien complejo o paradójico.

b) Los valores y las normas de una organización influyen en la manera de ser de sus miembros. De modo particular se consideran la honestidad, la responsabilidad, el respeto y la disciplina, dado a que el comportamiento individual depende de la interacción entre las características personales y el ambiente que lo rodea: la cultura organizacional puede potenciar, pero también limitar, el desarrollo de los individuos y de la propia institución. En particular, Montero (2019) plantea que los valores inspiran la razón de ser de cada institución, las normas vienen a ser los manuales de instrucciones para el comportamiento de la empresa y de las personas. Por lo tanto, toda organización con aspiraciones de excelencia debería tener comprendidos y sistematizados los valores y las ideas que constituyen el comportamiento motor de la empresa.

c) Interacciones: Las formas de relaciones entre los miembros de la organización, las prácticas usuales, las oportunidades frente a las autoridades que se establecen para crear confianza, cooperación y comunicación asertiva para evitar, confusiones, malentendidos, discriminaciones, rumores entre otros aspectos que intervienen en el contacto de autoridades y subordinados. Asimismo, se consideran para establecer armonía en las interacciones, el trabajo en equipo, la comunicación y las relaciones interpersonales.

d) Productividad: es el propósito de toda organización para lograr el éxito, por tanto, es imperante reconocer los perfiles profesionales en cada uno de los cargos que forma la jerarquía organizativa y las iniciativas que impulsan los cambios y transformaciones de las instituciones.

\section{Noción de Calidad Educativa}

Según la Organización de las Naciones Unidas para la Educación, la Ciencia y la Cultura (UNESCO) por sus siglas en ingles, a través de los pronunciamientos de 2010, afirma que la calidad de la educación es un tema preponderante que ha tenido un gran auge en los últimos años, y establece un marco de referencias para comprender, monitorear y mejorar la calidad educativa, asimismo dada a la diversidad de interpretaciones de la calidad que se hacen evidentes en las diferentes tradiciones, se requiere diálogos para definir la calidad y desarrollar enfoques que permitan monitorearla y mejorarla.

Esto significa alcanzar:

- Acuerdos generales sobre los objetivos y metas de la educación.

- Un marco de análisis de la calidad que permita especificar sus diversas dimensiones. 
- Un enfoque de medición que permite identificar y valorar las variables importantes.

- Un marco para la mejora que cubra de modo comprensivo los componentes vinculados del sistema educativo y que permita oportunidades para identificar los cambios y las reformas necesarias.

En efecto el mejoramiento de la calidad educativa ocupa un lugar importante en la política educativa colombiana, considerando señalamientos de organismos multilaterales y al respaldo de trabajo mancomunado de experiencias significativas en los procesos educativos colombianos (González \& Rincón, 2017).

De acuerdo al documento $\mathrm{N}^{\circ} 3$ Estándares Básicos de Competencias en Lenguaje, matemática, ciencias y ciudadanas de (Schmidt, 2006), la reflexión sobre la calidad de la educación nace de una fuerte crítica a la eficiencia interna de los sistemas educativos (acceso, retención, promoción, repitencia y deserción). Es hecho notorio que los resultados del modelo educativo vigente desde la década de los 60 y 70, no estaba efectivamente aportando al desarrollo social de las naciones y menos aún a satisfacer las necesidades de grandes sectores de la sociedad.

\section{Metodología}

El tipo de investigación es descriptiva, de acuerdo a González-Díaz and Hernández-Royett (2017) estas investigaciones se orientan a caracterizar, registrar, analizar e interpretar la naturaleza actual de las variables investigadas, tal como son observadas por el investigador. Al respecto, Hernández-Royett and González-Díaz (2016), definen este tipo de investigación como la manera de buscar, especificar propiedades, características y rasgos importantes de personas, grupos, comunidades o cualquier otro fenómeno que se someta a un análisis.

En este sentido, el estudio se realizó en la medida que se accede a la información, se describe la situación actual de las instituciones en el entorno social de sus rectores y su personal; en concreto, sus manifestaciones de la cultura organizacional: clima organizacional, los valores, las interacciones y la productividad, los cuales permiten alcanzar el objetivo propuesto.

Con respecto al diseño de la investigación, Hernández-Royett and González-Díaz (2016), lo define como una estrategia adoptada por el investigador para responder al problema planteado, es decir, el procedimiento concebido para obtener la información deseada. En vinculación a esta definición la investigación se enmarcó en un diseño no experimental, de campo y transeccional.

Para la selección de la muestra se realizó de forma intencionada, donde los elementos muestrales de la población son seleccionados bajo el estricto juicio personal del investigador, debido a la experiencia y conocimiento de este con respecto a las instituciones educativas de la zona. De aquí, que la muestra quedó constituida por 10 (diez) rectores y 40 (cuarenta) trabajadores que forman parte de personal docente, administrativo y obrero de las Instituciones Educativas Distritales de Santa Marta.

En cuanto a las técnicas e instrumento de recolección de la información, se realizó a través de la observación y la encuesta, aplicando un cuestionario conformado por 14 (catorce) items, que presenta una escala de selección de 4 (cuatro) alternativas (siempre, casi siempre, nunca y casi nunca), a lo que se les da el atributo de alta, mediana, baja y muy baja manifestación de la cultura organizacional de las instituciones estudiadas.

\section{Resultados}

Los datos recolectados fueron tabulados en una matriz de doble entrada a través de un procedimiento manual y presentados en números absolutos y porcentuales y representados en gráficos de línea que muestran las coincidencias y diferencias de las respuestas dadas entre los rectores y el personal que labora en las instituciones. El análisis de los resultados se presenta de forma cuantitativa y contrastado con autores. 


\subsection{Manifestaciones de la cultura organizacional}

De la información suministrada, se observa claramente en el Gráfico 1, que los rectores afirman en un 46,5\% que siempre se manifiesta un clima organizacional en sus instituciones, siendo solo un $20,63 \%$ la respuesta de siempre para el personal encuestado. Esto refleja que para la mayoría de los rectores hay una alta manifestación del clima organizacional. Contrariamente un $35,65 \%$ del personal considera que casi nunca se manifiesta el clima organizacional. Es posible notar que los valores coinciden en la respuesta de casi siempre menores al $30 \%$ de los encuestados. Por lo que se considera que se manifiesta medianamente el clima organizacional, en lo relativo a liderazgo, satisfacción, reconocimiento y ambiente físico de las escuelas objeto de estudio.

Gráfico 1

Manifestación del Clima Organizacional

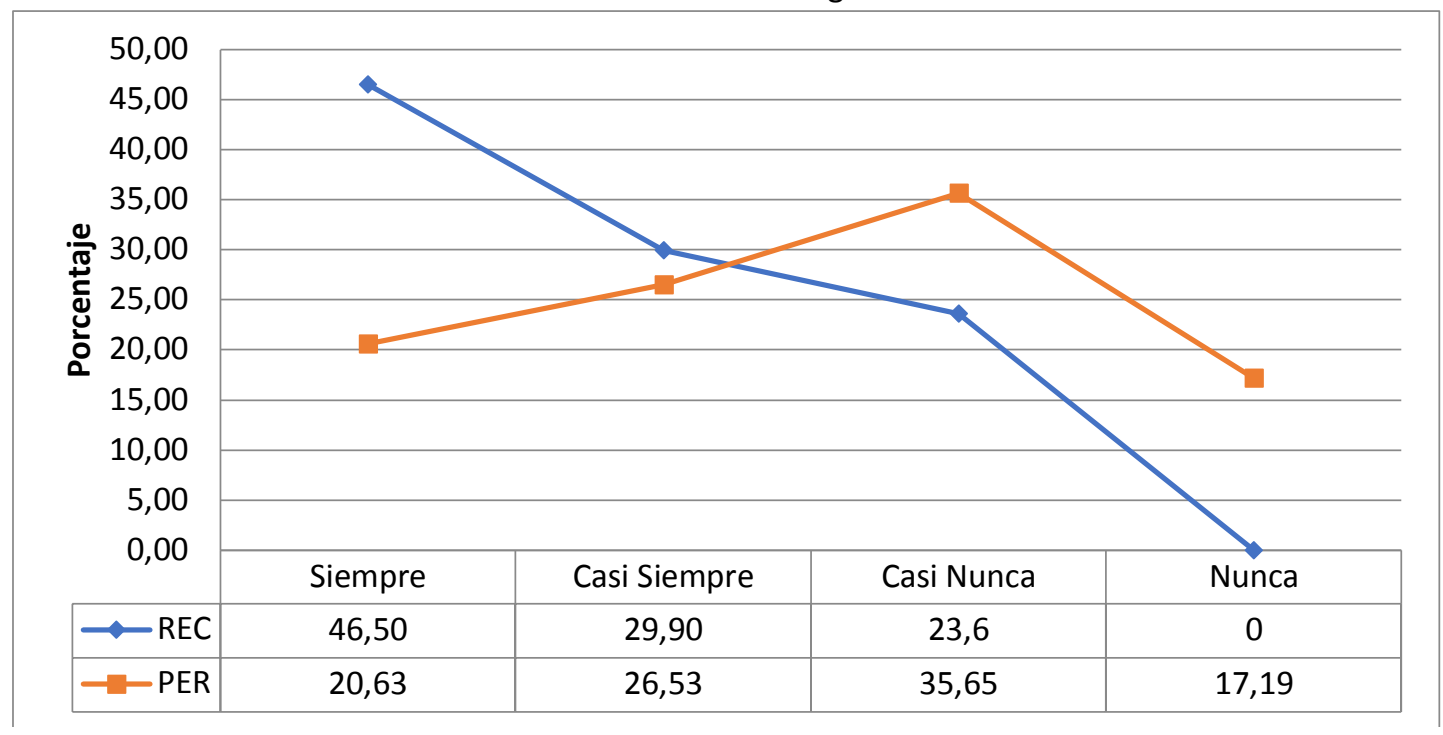

Fuente: Elaboración Propia (2020)

De acuerdo a Méndez and Hidalgo (2019), el clima organizacional es ambiente propio de la organización, producido y percibido por el individuo de acuerdo con las condiciones que encuentra en su proceso de interacción social y en la estructura organizacional. Se expresa por variables que orientan su creencia, percepción, grado de participación y actitud, determinando su comportamiento, satisfacción y nivel de eficiencia en el trabajo. No obstante, en algunas instituciones educativas de Colombia presentan conocimiento claro y consciente del clima organizacional, en consecuencia, se producen conflictos y debilidades en el trabajo.

\section{Valores}

En la información recabada para esta manifestación, se muestra en el Gráfico 2, que el 75,65\% de los rectores señalaron la alternativa siempre, seguido de un $34,35 \%$ como valor referido por el personal en la misma. No obstante, el 53,3\% del personal considera que se manifiestan los valores en su escuela, lo cual, quiere decir, que se plantea una tendencia de mediana hacia alta manifestación de los valores en las instituciones estudiadas, dado a los indicadores de honestidad, responsabilidad, respeto y disciplina.

Al respecto Carbonell Sebarroja (2019), señala que los valores son las creencias perdurables en un modo de conducta o estado final estando ligados a la personalidad, influyendo en el comportamiento. En este marco, los valores de una persona son concebidos como principios y postulados básicos que guían sus creencias, actitudes y comportamiento, así pues, se considera que los valores aun cuando se manifiestan en las instituciones educativas, se deben orientar de modo que reflejen con mayor equidad entre los miembros que las conforman. 
Gráfico 2

Manifestación de los Valores de la Organización

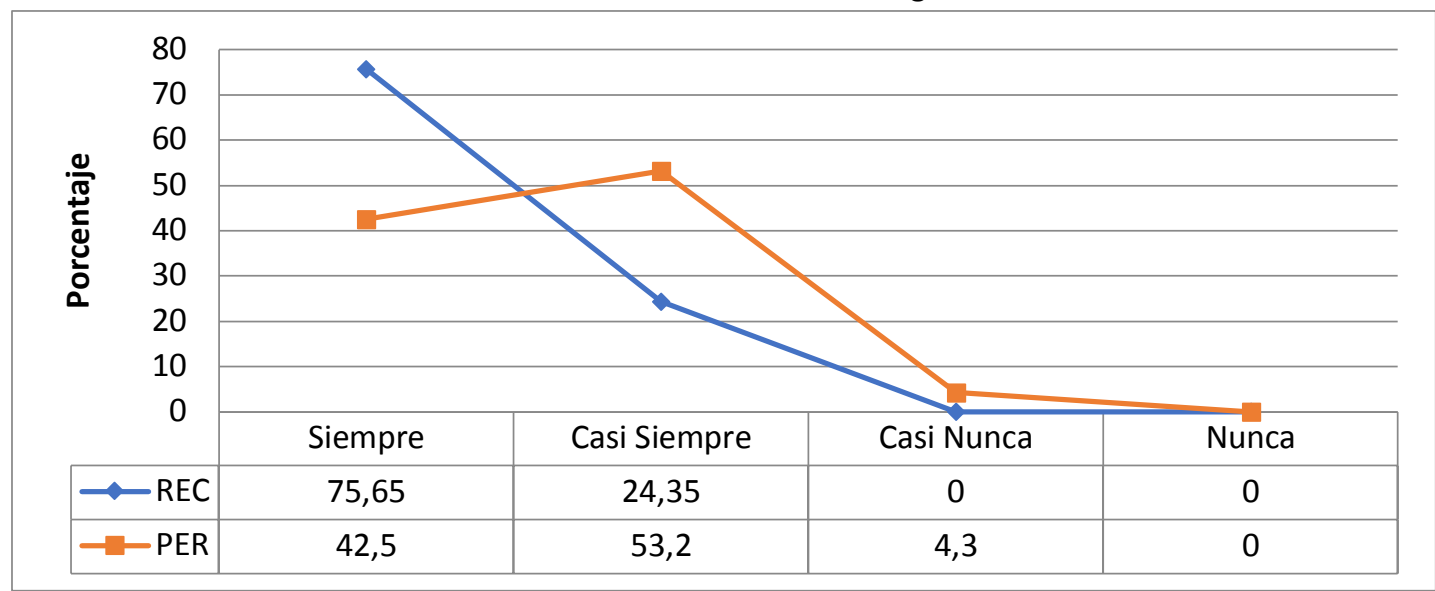

Fuente: Elaboración Propia (2020)

\section{Interacciones}

Los registros calculados para las interacciones, en la Gráfica 3, destacan que $58,4 \%$ de los rectores coinciden que siempre se manifiesta la interacción entre los miembros de su institución, acercándose al $40,4 \%$ de las respuestas obtenidas del personal.

Es notorio, además, el punto coincidente entre el resultado de las respuestas de los rectores y el personal en la alternativa casi siempre. Tal situación refleja evidentemente, que existe una tendencia de mediana a alta manifestación de los indicadores implícitos como son el trabajo en equipo, la comunicación y las relaciones interpersonales.

Gráfico 3

Manifestación de Interacciones

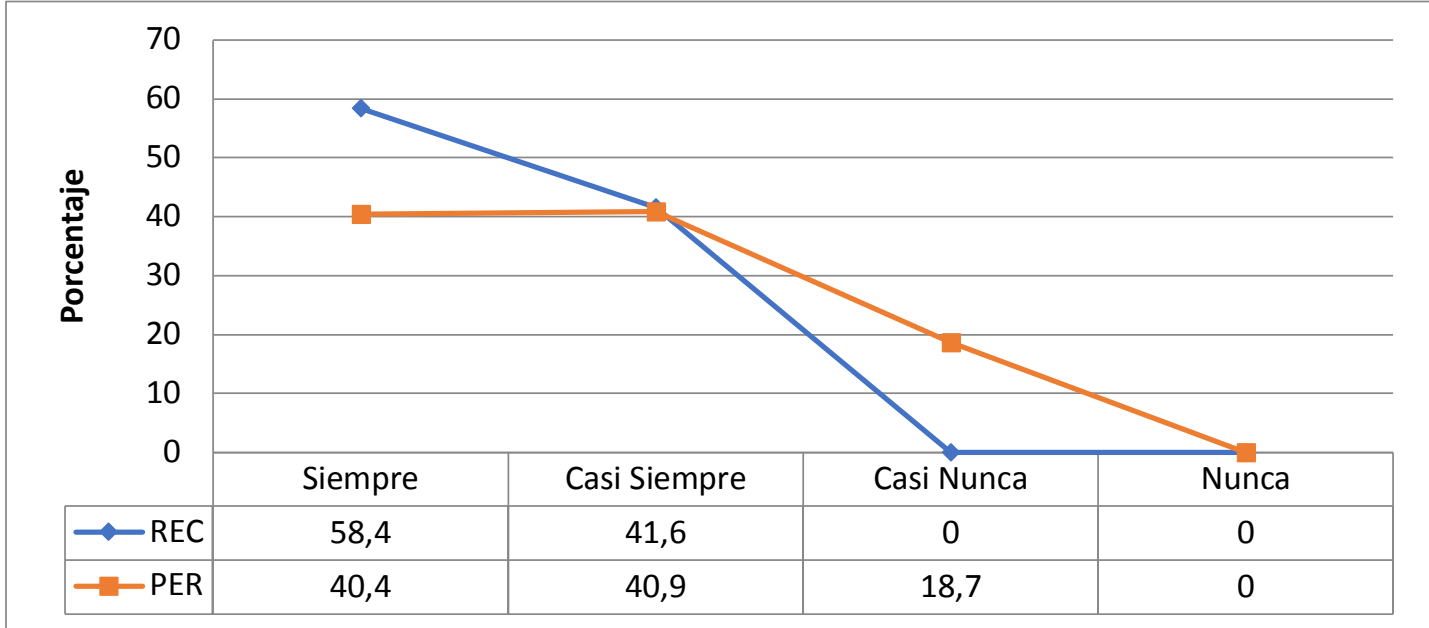

Fuente: Elaboración Propia (2020)

En conformidad con lo anterior Gallo Roldán and Ortiz Castaño (2019) consideran que existen un conjunto de paradigmas, que se forman a lo largo de la vida de la organización como resultado de las interacciones entre sus miembros, de estos con las estructuras, estrategias, sistemas, procesos, y de la organización con su entorno, a partir de las cuales se conforman un conjunto de referencias, que serán válidas en la medida que garanticen la 
eficiencia y la eficacia de la organización. De manera que se aplica en los mecanismos para llevar los procesos de gestión para la calidad educativa.

\section{Productividad}

Como se indica en el Gráfico 4, el 73,3\% de los rectores y el 55,5\% del personal señalan que siempre se apunta hacia la productividad en lo que corresponde al perfil profesional de sus funcionarios y las iniciativas que se emprenden en las escuelas. Este resultado apunta una alta tendencia en la cultura organizacional sana en las instituciones educativa distritales de Santa Marta, situación considerada para desarrollar una buena gestión de la calidad educativa e impulsar los valores promovidos por los entes gubernamentales.

Gráfica 4

Manifestación de la Productividad

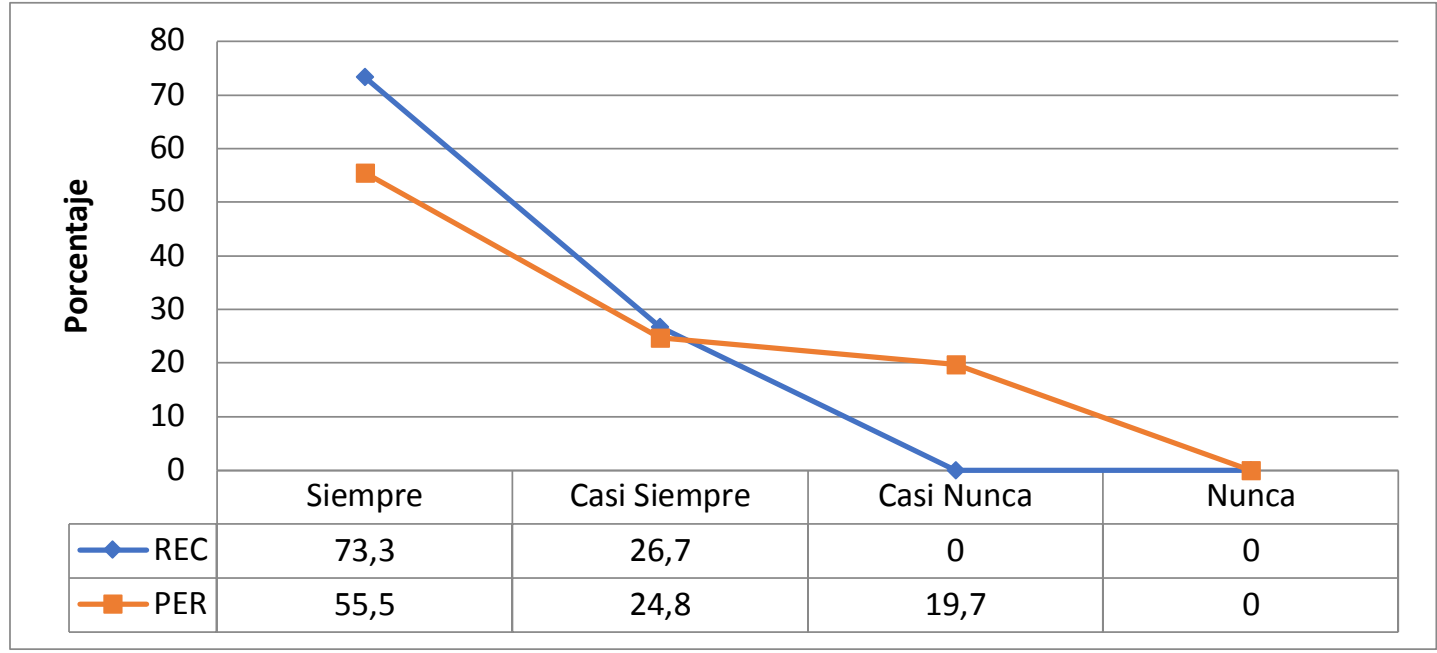

Fuente: Elaboración Propia (2020)

En torno a esta consideración López (2019), explica que las organizaciones actualmente se desempeñan en entornos de alta competitividad y creciente globalización, caracterizados por constantes cambios a ritmos muy acelerados, con la necesidad de integrar una creciente diversidad de perspectivas y enfoques y un diseño organizacional cada vez más plano y menos jerárquico.

Asimismo, es notable los resultados obtenidos, y en este sentido se aplica lo referido por Huamán Fernádez (2019), al señalar que al trabajar sobre los elementos que conforman la cultura organizacional de un centro educativo, brindaría la oportunidad a los miembros que la constituyen de una mejor gestión en el aspecto educativo. Es cierto que una organización eficaz es determinante en la obtención de mejores resultados de una escuela, lo cual quiere decir que debe haber una cultura organizacional para permitir las mejores condiciones de trabajo y brindar el mejor producto, por lo tanto, debe existir alguien que sepa dirigir y organizar los recursos, quien indudablemente podría ser el director en conjunto con el personal docente de la escuela.

\section{Conclusiones}

Los resultados ilustran la complejidad del proceso escolar y ameritan estudios más dedicados al tema de la cultura organizacional que denotan debilidades, fortalezas y logros como se han observado en los resultados de la investigación. Se ve con preocupación la discordancia entre rectores y el personal docente, administrativo y obrero involucrados en la investigación acerca de cómo es su perspectiva en cuanto a la manera como se manifiesta el clima organizacional, considerándose el liderazgo, satisfacción, reconocimiento y ambiente físico, en donde es necesario profundizar en estudios que estén orientados a la reflexión y convicción de los actores para responder a un mejor desenvolvimiento y desempeño. 
Por otro lado, se resalta que las manifestaciones resultantes de los valores y las interacciones caracterizan mayoritariamente en la cultura organizacional de las instituciones educativas, considerándose además la productividad, para ejecución de acciones que contribuyan al logro de las metas y objetivos institucionales en virtud de las exigencias actuales dirigidas a mejorar la gestión para alcanzar la calidad de la educación en Colombia.

De aquí que los elementos para asegurar el éxito y la existencia de las organizaciones educativas, se centra en generar consenso entre las actitudes, motivaciones, expectativas, aspiraciones, enmarcado en el proyecto nacional de todos por un nuevo país en equidad paz y educación.

\section{Referencias bibliográficas}

Álvarez, C. E. M. (2019). Elementos para la relación entre cultura organizacional y estrategia. Universidad \& Empresa, 21(37), 136-169. https://dialnet.unirioja.es/servlet/articulo?codigo=7084468

Arboleda, M. L. O., \& Díaz, R. R. G. (2017). El carácter axiológico del proceso educativo en Colombia. Centros: Revista Científica Universitaria, 6(2), 1-17. https://revistas.up.ac.pa/index.php/centros/article/view/3

Burke, P. (2020). Hibridismo cultural (Vol. 353): Ediciones Akal. https://bit.ly/2TNIlxG

Carbonell Sebarroja, J. (2019). Escuela y entorno. uri: https://repositorio. idep. edu. co/handle/001/196.

Carrascal, A. I. O., \& Giraldo, J. J. (2019). Minería de datos educativos: Análisis del desempeño de estudiantes de ingeniería en las pruebas SABER-PRO. Revista Politécnica, 15(29), 128-140. https://revistas.elpoli.edu.co/index.php/pol/article/view/1499

Carrillo-Punina, Á. P. (2019). Cultura organizacional y desempeño financiero en las cooperativas de ahorro y crédito ecuatorianas. Universidad Nacional de La Plata. http://sedici.unlp.edu.ar/handle/10915/75026

Díaz, R. R. G., \& Ramos, D. E. C. (2019). Crecimiento financiero en las entidades del sector cooperativo a través de la bolsa de valores de Colombia. Centros: Revista Científica Universitaria, 8(1), 127-143. http://portal.amelica.org/ameli/jatsRepo/228/2281003009/2281003009.pdf

Echeverría, B. (2019). Definición de la cultura: Fondo de Cultura Económica. https://bit.ly/3869sMX

Freire, S. N. B., Jácome, O. H., \& Yandún, P. F. (2019). Relación entre rendimiento académico y asistencia como factores de promoción estudiantil. Cátedra, 2(1), 44-59. http://200.12.169.32/index.php/CATEDRA/article/view/1552

Gallo Roldán, S. M., \& Ortiz Castaño, J. (2019). La interacción entre agentes educativos y familias participantes en el Centro Infantil Hermanas Misioneras de la Comunidad Cristiana: Una mirada desde la gestión del conocimiento en el marco del Programa Buen Comienzo de la Secretaría de Educación Alcaldía de Medellín. http://ayura.udea.edu.co:8080/jspui/handle/123456789/3596

García, M. D. G. (2020). Dirección escolar con enfoque de liderazgo carismático. Perfiles de un líder educativo. Dominio de las Ciencias, 6(2), 718-739. https://dialnet.unirioja.es/servlet/articulo?codigo=7398464

González-Díaz, R. R., \& Hernández-Royett, J. (2017). Diseños de investigación cuantitativos aplicados en las ciencias de la administración y gestión. Globalciencia, 3(1), 15-27. https://globalciencia.com/index.php/globcien/article/view/11

González, R., \& Rincón, M. (2017). Análisis de la producción escrita de niños con síntomas de dislexia a partir de un enfoque Lingüístico-Cognitivo. Revista Oratores(4). https://revistas.umecit.edu.pa/index.php/oratores/article/view/76 
Hernández-Royett, J., \& González-Díaz, R. R. (2016). Enfoques de investigación en la contabilidad. Estrategia, 2(1), 87-100. http://estrategia.unisinu.edu.co/index.php/estrat/article/view/29

Huamán Fernádez, V. E. (2019). La Gestión del Capital Humano y Productividad Laboral del Personal de una Entidad Educativa Privada, Arequipa 2016. http://repositorio.uancv.edu.pe/handle/UANCV/2739

LA UNESCO, S. L. D. (2010). Declaración Universal de la UNESCO sobre la diversidad cultural. PRAXIS, $64,65$. https://www.revistas.una.ac.cr/index.php/praxis/article/download/4080/3919/0

Leonard, Y. G., Pérez, R. L., \& Domínguez, M. D. (2020). La cultura organizacional en las entidades turísticas del destino la habana en la gestión de la innovación. ConcienciaDigital, 3(1), 36-49. http://www.cienciadigital.org/revistacienciadigital2/index.php/ConcienciaDigital/article/view/997

López, S. (2019). Eficiencia y calidad en la actividad educativa: Una aplicación del índice de productividad de Metafrontera Malmquist-Luenberger. https://www.redalyc.org/pdf/904/90452745010.pdf

Maldonado, C., \& del Pilar, M. (2019). Gestión pedagógica y calidad educativa en la Institución Educativa 3063 Patricia Natividad Sánchez de Independencia. http://200.60.81.165/handle/UNE/3590

Mead, M. (2019). Cultura y Compromiso: estudios sobre la ruptura generacional: Editorial Gedisa. https://bit.ly/37ZdAOQ

Méndez, C. G. C., \& Hidalgo, J. F. O. (2019). Responsabilidad social y clima organizacional en la universidad autónoma de NAYARIT. Proyecciones(13), 002-002. https://revistas.unlp.edu.ar/proyecciones/article/view/9316

Montero, E. C. (2019). Desarrollo de valores organizacionales para la innovación social. Una mirada desde el emprendimiento en el contexto cubano actual/Articulating Organizational Values for Social Innovation. An Entrepreneurship-Based View Thereon in Today's Cuban Context. Revista Economía y Desarrollo (Impresa), 161(1). http://scielo.sld.cu/scielo.php?script=sci_arttext\&pid=S0252-85842019000100003

Ovalles-Toledo, L. V., Martínez, V. A. S., Arrieta, J. A. B., \& Aguas, E. J. S. (2016). Ética en los procesos gerenciales de las Pymes en Colombia. Estrategia, 2(1), 20-32. http://estrategia.unisinu.edu.co/index.php/estrat/article/view/30

Schmidt, Q. (2006). Estándares básicos de competencias en lenguaje, matemáticas, ciencias y ciudadanas: guía sobre lo que los estudiantes deben saber y saber hacer con lo que aprenden [1: Ministerio. https://edumedia-depot.gei.de/bitstream/handle/11163/1921/788071114_2006_A.pdf?sequence=6

Steiner, G. (2020). En el castillo de Barba Azul: aproximación a un nuevo concepto de cultura: Editorial Gedisa. https://bit.ly/2TL1PTK

Touriñán López, J. M. (2019). La relación educativa es un concepto con significado propio que requiere concordancia entre valores y sentimientos en cada interacción. Sophia, colección de Filosofía de la Educación(26), 223-279. http://scielo.senescyt.gob.ec/scielo.php?script=sci_arttext\&pid=S139086262019000100223

Valdeyglesias, C., Gerty, J., Chávez Muñoz, K. M., \& Ortiz Castillo, A. N. (2019). Apreciación inicial de la evolución e impacto de las manifestaciones de la cultura y percepción del clima laboral en una empresa local familiar, tras un proceso de adquisición por una empresa transnacional. https://repositorioacademico.upc.edu.pe/handle/10757/625936

Velazquez Valadez, G. (2019). Liderazgo Empático "Un modelo de liderazgo para las Organizaciones Mexicanas". http://repositorio.lasalle.mx/handle/lasalle/975?locale-attribute=es

Esta obra está bajo una Licencia Creative Commons Attribución-NoCommercial 4.0 International 